\title{
Challenges and Opportunities to Improve Tuberculosis Screening among Immigrant Plantation Workers in Sabah, Malaysia
}

\author{
Maini Sabait ${ }^{1}$, Wan Mohd Zahiruddin Wan Mohammad², Mohamed Rusli Abdullah ${ }^{3}$, Julia Omar ${ }^{2}$ \\ ${ }^{1}$ State Health Department of Sabah, Kota Kinabalu, Sabah, Malaysia \\ ${ }^{2}$ School of Medical Sciences, Universiti Sains Malaysia, Malaysia \\ ${ }^{3}$ International Medical School, Management and Science University, Malaysia
}

\begin{tabular}{|c|c|}
\hline Article Info & ABSTRACT \\
\hline Article history: & Tuberculosis (TB) among immigrants has substantial contribution to the TB \\
\hline Received Jan 12, 2015 & for TB disease among immigrant plantation workers in Sabah, Malaysia. \\
\hline Revised Aug 21, 2015 & This was a prospective cohort study involving 482 legal immigrant workers \\
\hline Accepted Jan 26, 2016 & $\begin{array}{l}\text { aged } 18 \text { years and above, consented and available at study sites during the } \\
\text { study period. Workers with previous history of TB or currently on TB }\end{array}$ \\
\hline Keyword: & $\begin{array}{l}\text { treatment were excluded from participation. Symptom based questionnalre } \\
\text { was administered along with both chest radiograph and sputum samples }\end{array}$ \\
\hline Case detection & creened, there was no case of active TB detected among the $44(9.1 \%)$ \\
\hline Immigrant workers & symptomatics participants. Finding of low TB yield in this study was rather \\
\hline Screening & unexpected but this indicates the real challenges for the local health authority \\
\hline Tuberculosis & $\begin{array}{l}\text { to come out with more cost effective screening programs, including reducing } \\
\text { stigma, in active TB screening among migrant population. }\end{array}$ \\
\hline
\end{tabular}

Copyright (c) 2016 Institute of Advanced Engineering and Science. All rights reserved.

\section{Corresponding Author:}

Wan Mohd Zahiruddin Wan Mohammad, Department of Community Medicine, School of Medical Sciences, Universiti Sains Malaysia, 16150 Kubang Kerian Kelantan Malaysia. Email: drzahir@usm.my

\section{INTRODUCTION}

Sabah is one of the thirteen states in Malaysia with high tuberculosis (TB) burden despite the country status as intermediate burden country. Increasing TB case notifications of more than 100 case notification per 100,000 population despite the overall country decrease was observed [1]. This increase has been associated with the immigrant entry from TB endemic neighbouring countries [2]. Filipino immigrants (country of origin from the Philippines) contribute to about a third of the total number of TB cases reported in Sabah [3]. TB among immigrants has been increasingly challenging the public health system among other issues related to immigrants. Immigrants have disproportionately higher prevalence of TB compared to the native population due to their migration status. They are socially disadvantaged, living in crowded housing where they are highly likely to encounter individual with TB and poor health access [4]. Additionally, immigrants came from countries with high TB incidence as well as latent tuberculosis infection (LTBI) thus the increased risk of active TB [5].

Upscaling TB case detection and prompt treatment of identified new TB cases remains top priority in TB control in Sabah [1]. In conjunction to this effort, screening immigrants who are thought to be at high risk for TB disease were carried out. Screening for TB disease are performed as a tool in TB burden measurement, monitoring TB control programme performance as well as improving detection of TB resulting in earlier detection and treatment and hence cutting short the TB transmission in the community. 
Screening tools that were usually utilised in screening are questionnaires on TB symptoms, chest radiography, sputum microscopy, sputum culture, or combinations of these tools [6]. However, screening in practice was variable in terms of approaches and screening tools used [7].

In this study, we conducted the first field screening for active TB among legal immigrants working in Sabah palm oil plantation. This paper describes the yield of screening, issues identified and recommendations that may be applicable for future screening activity.

\section{RESEARCH METHOD}

This study was a prospective cohort study conducted in a palm oil plantation at Sandakan, a district with the second highest TB notification in Sabah. In May 2013, 482 legal immigrant workers aged 18 years and above were recruited to participate in the study. Immigrant workers with previous history of TB or was currently on TB treatment were excluded from participation. Information regarding the study was given and informed consent was sought from interested workers. All the sessions were conducted in Malay language as the workers were proficient in the language.

After obtaining consent, the workers were interviewed based on a standard proforma. This included socio-demographic data and migration history while eliciting symptoms compatible with TB. Presence of BCG scar was also examined. A screening question on presence of cough was asked in order to separate coughing participant from the rest as well as given face mask. Workers with TB symptoms had their sputum samples collected. Two spot sputum samples and one morning samples were considered adequate. The first spot sputum samples were taken before the interview while the second one was taken post interview. The morning sample was taken the on following day. Participants with TB symptoms were also instructed to have their chest radiograph examination taken at the nearest district hospital. The plantation management provided the transportation for the participants for this matter.

Data were analysed using SPSS version 22 (IBM Corporation, Somers, NY, USA). Categorical variables were presented as frequencies and percentage (\%) while numerical variables were presented using mean and standard deviation (SD). The study protocol was reviewed and approved by the Universiti Sains Malaysia Human Research Ethics Committee (USMKK/PPP/JEPeM 265.3(7)). Participation was completely voluntary and workers could withdraw at any time. The workers were not disadvantaged by refusal to participate in the study or any component of the study. Confidentiality was kept at all times.

\section{RESULTS AND ANALYSIS}

A total of 482 plantation workers were involved as the study participants. The result describes the analysis on the baseline screenings performed in this study.

\subsection{Socio Demographic}

Table 1 shows the socio-demographic profile of the study participants. Four hundreds and sixty five (96.5\%) plantation workers were Indonesian immigrants. The fact that majority of the study participants were of Indonesian origin could be one of the explanations for the yield of TB disease screening in this study, and whether appropriate inference on the TB risk and incidence can be made to all immigrants in the state.

Table 1. Socio-demographic profile of study participants $(n=482)$

\begin{tabular}{lc}
\hline \multicolumn{1}{c}{ Variables } & $\mathbf{n}(\mathbf{\%})$ \\
\hline Age categories (years) & $165(34.2)$ \\
$18-29$ & $189(39.2)$ \\
$30-39$ & $113(23.4)$ \\
$40-49$ & $12(2.5)$ \\
$50-59$ & $3(0.6)$ \\
$60-65$ & \\
Sex & $260(53.9)$ \\
Male & $222(46.1)$ \\
Female & \\
Country of origin & $465(96.5)$ \\
Indonesia & $17(3.5)$ \\
Philipines & $11.7(5.99)$ \\
Time since entry to Sabah (mean, SD) & $21.8(7.01)$ \\
Age at entry to Sabah (mean, SD) & $25(5.2)$ \\
History of TB contact & $131(7.2)$ \\
Presence of BCG Scar & \\
\hline
\end{tabular}

IJPHS Vol. 5, No. 1, March 2016: $41-45$ 
The study also noted that only $5.2 \%$ of the study participants have history of contact with TB patient. However, it has been shown in previous studies that having TB disease or associated with anyone with TB could lead to stigma [8]. Since we relied on participants to supply the information, we could not ascertain whether some participants chose not to reveal their association to a TB patient. Furthermore, participants may not know for sure whether the symptoms of their colleague or family members were really TB disease. Most active case detection relied on history of contact before deciding whether or not to investigate them further [9],[10]. The implication of this finding was health care provider may be prevented from assisting them in obtaining prompt and proper TB treatment.

\subsection{Tuberculosis Symptoms}

The screening in this study was successful in identifying 44 (9.1\%) symptomatic participants. Table 2 shows the proportion of participants having TB symptoms. Cough was the most common TB compatible symptoms identified among the participants. Out of 44 participants with cough, only 3 (6.8\%) of the participants had seek medical attention for their symptoms. One of the participants has the longest cough duration of 3 months without ever seeking medical attention. It should be borne in mind that there might be a number of participants who did not reveal presence of TB symptoms. However, it should be remembered that cough may be regarded as normal among smokers [11]. In previous studies, stigma related to TB diagnosis, fear of deportation, being placed at disadvantage, and discrimination associated with TB diagnosis was noted. Thus, fear of being diagnosed with TB disease might lead to avoidance from seeking health care [8].

\begin{tabular}{lc} 
Table 2. TB Symptoms among the study participants (n=44) \\
\hline \multicolumn{1}{c}{ Variables } & $\mathbf{n}(\%)$ \\
\hline Any cough & $44(9.1)$ \\
Cough more than 7 days & $13(29.5)$ \\
Duration of cough (mean,SD & $0.69(4.93)$ \\
Productive with sputum & $24(5 \%)$ \\
Haemoptysis & $0(0.0)$ \\
Fever & $9(1.9)$ \\
Night sweats & $3(0.4)$ \\
Loss of weight & $3(0.6)$ \\
Lymph node swelling & $0(0.0)$ \\
\hline
\end{tabular}

Thirthy eight (86.4\%) the symptomatics participants submitted adequate sputum sample for sputum microscopy examination. However, none of the sputum microscopy samples were found to be positive. Chest radiograph done for all the participants with symptoms compatible with TB symptoms also did not have evidence of active TB disease. Although chest radiograph as a screening tool was well established, however of concern were the fact that immigrant population have preponderance to extrapulmonary TB [12]. Hence the limited usefulness of chest radiograph in detecting extra pulmonary TB. This study was also limited by the fact that the focus was more on detection of pulmonary tuberculosis. It is also possible that some cases could have been missed by chest radiograph [13] as the findings could have lagged behind the clinical pictures [11].

Another possible reason was that the participants were mostly non recent immigrants who have stayed in Sabah for more than a decade (an average of nearly 12 years), hence the lower risk of getting TB as shown by finding from Frothingham et al. [14]. However, more recent studies showed the risk for developing active TB remain high even after many years post migration [12],[15],[16]. The findings might also imply that probably better living conditions of the labour immigrants in the state plantations as evidence has shown that a significant proportion of TB among migrants is actually reactivated or newly acquired as a result of the poor conditions through which migrants travel and then work and live [17]. Apart from that, the study findings might also indicate the rigorous screening policies designed to prevent the entry of migrants with active TB have been done [18], here by both origin countries (Indonnesia and the Philippinse) and also the receiving country Malaysia.

As the previous study by Dony et al. [3] which showed a third of the TB cases in Sabah were Filipino immigrants, it is thus important to consider appropriate representative samples of immigrants to Sabah in the future study particularly those from the Phillipines. On the other hand, this finding could serve to inform us that the current medical examination for immigrant workers in this setting has performed well. The participants in this study have had multiple annual medical examinations. Hence, they could have 
benefitted from the regular workers medical examination resulting in none of the participants diagnosed with active TB during the study period.

Findings of low yield in TB screening among immigrants has been shown in other studies [19]. However, this should not detain the health provider from continuing to screen immigrants as it was shown that earlier case detection, reduced admission to hospital, duration of symptoms were shortened and hence decrease TB transmission could result from screening programmes [20]. Furthermore, immigrant populations have an elevated risk of TB even after many years of settling into a new country [12],[21]. Therefore, it is important that surveillance for active TB should be continued vigilantly as for recent immigrants as the WHO’s Western Pacific Region, which include countries like Malaysia and the Philippines, bears nearly onefifth of the gobal TB burden accompanied by the alarmingly increasing burden of MDR-TB [22].

\section{CONCLUSION}

This study showed that TB screening among immigrants could be performed in the field setting especially at their work and living places. However, it should be noted that finding of low TB yield among immigrant workers in this study was rather unexpected given higher background TB prevalence in their origin country. Many possible reasons have been explained but this may become the real challenges among the local health authority of the receiving country like Malaysia to come out with better approaches and more cost effective TB screening programs among migrants. Importantly, advocacy and public education efforts among the government and other stakeholders, including employers, on the importance of ensuring access to TB screening and care for migrant populations need to be strengthened as well.

\section{ACKNOWLEDGEMENTS}

The authors would like to firstly thank Sawit Kinabalu Sdn.Bhd for allowing the use their facilities to conduct this study. We would also like to acknowledge the support and assistance from the State Health Department of Sabah, Dr Aziah Daud, Dr Aziah Ismail, Dr Ahmad Filza and Dr Noor Khalili, in the process of completing this research. The study was funded by Ministry of Education's Long Term Research Grant (LRGS) and Universiti Sains Malaysia’s Short Term Research (no. 304/PPSP/61312117) grant schemes. The funding body has no involvement in the design, data collection, analysis and interpretation of data, as well as in the preparation of this manuscript.

\section{REFERENCES}

[1] Avoi, R., “Tuberculosis Control Strategic Plan in Sabah 2011-2015’, Kota Kinabalu, Sabah, 2013.

[2] Aziah, AM., "Tuberculosis in Malaysia: combating the old nemesis”, Med J Malaysia, vol/issue: 59(1), pp. 1-3, 2004.

[3] Dony, JF., J. Ahmad, Y. Khen Tiong, “Epidemiology of tuberculosis and leprosy, Sabah, Malaysia”, Tuberculosis (Edinb), vol/issue: 84(1-2), pp. 8-18, 2004.

[4] Lonnroth, K., E. Jaramillo, BG. Williams, C. Dye, M. Raviglione, "Drivers of tuberculosis epidemics: the role of risk factors and social determinants”, Soc Sci Med, vol/issue: 68(12), pp. 2240-6, 2009.

[5] Greenaway, C., A. Sandoe, B. Vissandjee, I. Kitai, D. Gruner, W. Wobeser, K. Pottie, E. Ueffing, D. Menzies, K. Schwartzman, "Tuberculosis: evidence review for newly arriving immigrants and refugees", CMAJ, vol/issue: 183(12), pp. E939-51, 2011.

[6] Mor, Z., A. Leventhal, AH. Diacon, R. Finger, OD. Schoch, "Tuberculosis screening in immigrants from highprevalence countries: interview first or chest radiograph first? A pro/con debate”, Respirology, vol/issue: 18(3), pp. 432-8, 2013.

[7] Coker, R., A. Bell, R. Pitman, JP. Zellweger, E. Heldal, A. Hayward, A. Skulberg, G. Bothamley, R. Whitfield, G. de Vries, JM. Watson, “Tuberculosis screening in migrants in selected European countries shows wide disparities”, Eur Respir J, vol/issue: 27(4), pp. 801-7, 2006.

[8] Frothingham, R., JE. Stout, CD. Hamilton, "Current issues in global tuberculosis control”, Int J Infect Dis, vol/issue: 9(6), pp. 297-311, 2005.

[9] Fox, GJ., CC. Dobler, GB. Marks, “Active case finding in contacts of people with tuberculosis”, Cochrane Database Syst Rev, vol. 9, pp. Cd008477, 2011.

[10] Del Portillo-Mustieles, EC., R. Laniado-Laborin, “Active Case Finding of Pulmonary Tuberculosis through Screening of Respiratory Symptomatics Using Sputum Microscopy: Is It Time to Change the Paradigm?”, Tuberc Res Treat, vol. 2013, pp. 312824, 2013.

[11] Mathez, C., Y. Bangala, P. Bady, JP. Zellweger, “Active screening for pulmonary tuberculosis among immigrants by chest X-ray at the Swiss border”, Swiss Med Wkly, vol/issue: 137(45-46), pp. 649-54, 2007.

[12] Farah, MG., HE. Meyer, R. Selmer, E. Heldal, G. Bjune, "Long-term risk of tuberculosis among immigrants in Norway”, Int J Epidemiol, vol/issue: 34(5), pp. 1005-11, 2005. 
[13] Pepper, T., P. Joseph, C. Mwenya, GS. McKee, A. Haushalter, A. Carter, J. Warkentin, DW. Haas, TR. Sterling, "Normal chest radiography in pulmonary tuberculosis: implications for obtaining respiratory specimen cultures", Int J Tuberc Lung Dis, vol/issue: 12(4), pp. 397-403, 2008.

[14] McKenna, MT., E. McCray, I. Onorato, "The epidemiology of tuberculosis among foreign-born persons in the United States, 1986 to 1993”, N Engl J Med, vol/issue: 332(16), pp. 1071-6, 1995.

[15] Lillebaek, T., AB. Andersen, A. Dirksen, E. Smith, LT. Skovgaard, A. Kok-Jensen, "Persistent high incidence of tuberculosis in immigrants in a low-incidence country”, Emerg Infect Dis, vol/issue: 8(7), pp. 679-84, 2002.

[16] McPherson, ME., H. Kelly, MS. Patel, D. Leslie, "Persistent risk of tuberculosis in migrants a decade after arrival in Australia”, Med J Aust, vol/issue: 188(9), pp. 528-31, 2008.

[17] Ho, MJ., "Migratory journeys and tuberculosis risk”, Med Anthropol Q, vol/issue: 17(4), pp. 442-58, 2003.

[18] Zimmerman, C., L. Kiss, M. Hossain, "Migration and health: a framework for 21st century policy-making”, PLoS Med, vol/issue: 8(5), pp. e1001034, 2011.

[19] Arshad S., Bavan L., Gajari K., Paget SN., Baussano I., "Active screening at entry for tuberculosis among new immigrants : a systematic review and meta analysis”, Eur Respir J, vol. 35, pp. 1336-1345, 2010.

[20] Verver, S., R. Bwire, MW. Borgdorff, "Screening for pulmonary tuberculosis among immigrants: estimated effect on severity of disease and duration of infectiousness”, Int J Tuberc Lung Dis, vol/issue: 5(5), pp. 419-25, 2001.

[21] Cowie, RL., JW. Sharpe, "Tuberculosis among immigrants: interval from arrival in Canada to diagnosis. A 5-year study in southern Alberta”, CMAJ, vol/issue: 158(5), pp. 599-602, 1998.

[22] WHO, "Consultation on Tuberculosis and Migration in the Western Pacific Region”, Manila, Philippines, 2013.

\section{BIOGRAPHIES OF AUTHORS}
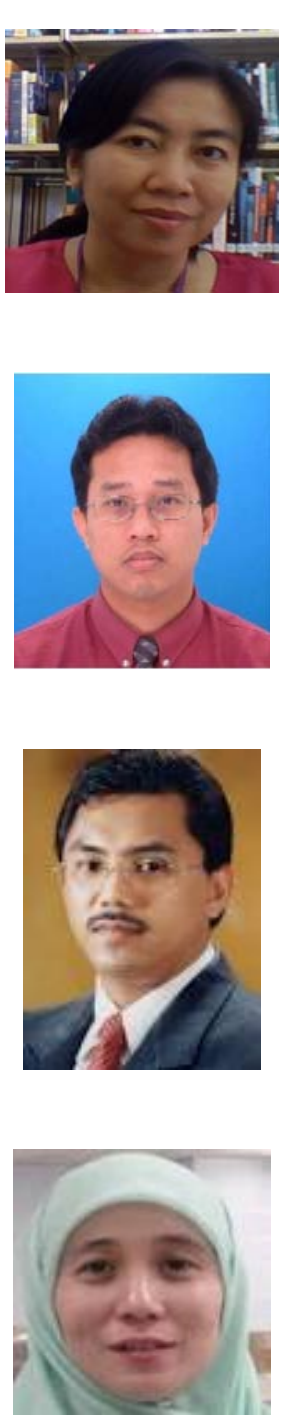

Maini Sabait (MD, MPH) is a doctorate in public health (Epidemiology) graduated from Universiti Sains Malaysia in 2015. She is currently posted and working in the Sabah State Health Department, Malaysia.
Wan Mohamed Zahiruddin Wan Mohammad (MD, M.Comm. Med (Epid. \& Biostats) is an Associate Professor in community medicine in Universiti Sains Malaysia. His research interests are tuberculosis, HIV, leptospirosis and communicable disease epidemiology. He is actively involved in conducting workshop in research methodology and medical statistics as well as consultancy in evaluation of public health programs.
Mohamed Rusli Abdullah (MD, MPH, and Phd) is a Dean and Professor in Epidemiology (Communicable Diseases and Tropical Medicine) with subspecialisation in sports medicine. He is an active figure in epidemiological study and has been involved in various research projects in public health in Malaysia.
Julia Omar (MD, MPath) is a pathologist with a vast experience in the field of chemical pathology. She is currently the head of department of Chemical Pathology in Universiti Sains Malaysia. 